\title{
A Microilluminator for the Study of the Infrared Spectra of Small Samples at Low Temperatures* ${ }^{*}$
}

\author{
Richard M. Badger and Roger Newman \\ Gates and Crellin Laboratories of Chemistry, California Institute of Technology, Pasadena, California
}

(Received April 17, 1951)

\begin{abstract}
An instrument is described which combines the functions of a reflecting microscope and a low temperature cell. It permits infrared absorption spectra to be obtained on single microcrystals at low temperatures, using polarized radiation.
\end{abstract}

$S^{\mathrm{s}}$ EVERAL investigators have recently described the use of a reflecting microscope, in conjunction with a spectrometer, to obtain infrared spectra on extremely small samples. ${ }^{1}$ Other workers have given details of special absorption cells with which one may obtain the infrared spectra of solids at low temperatures. ${ }^{2,3}$ In the following we describe an instrument which combines the functions of these two devices. It was designed primarily to permit low temperature studies to be made on single microcrystals with polarized infrared radiation.

In this laboratory we have had in operation for some time a reflecting microscope or microilluminator, the mounting of which is somewhat unconventional. The construction is such that when low temperature studies became desirable, it could with little trouble be modified to permit evacuation of the optical path, and to provide cooling of the sample.

As may be seen by reference to Fig. 1 the optical system is contained in a cylindrical case having four symmetrically located side arms. One of these serves as connection to the vacuum system and another as support for a Dewar vessel carrying the sample holder. At front and rear the housing is closed by plates (not shown) which carry appropriate infrared transmitting windows. One of these is held in place by a short housing, containing a silver chloride plate polarizer, ${ }^{4,5}$ which screws on the front end of the instrument. At the rear the illuminator is bolted to a rather massive plate which is rigidly attached to the spectrometer. This plate both serves as support and carries the exit window. Vacuum seals between the illuminator casing and its attachments are made with " $O$ " rings.

Our microilluminator was designed primarily for use with the Beckman IR-2 spectrometer, and is designed for insertion in a parallel beam. When used with this instrument it takes the place of the liquid cell compartment. This substitution can be conveniently made

* Contribution No. 1559.

$\dagger$ The instrument here described was developed in connection with a research program supported by the ONR under Contract N6-ori-102, VI.

1 To the best of our knowledge the use of this procedure was first described by Barer, Cole, and Thompson, Nature 163, 198 (1949).

2 E. L. Wagner and D. F. Hornig, J. Chem. Phys. 18, 296 (1950).

${ }^{3}$ A. Walsh and J. B. Willis, J. Chem. Phys. 18, 552 (1950).

${ }^{4}$ R. Newman and R. S. Halford, Rev. Sci. Instr. 19, 270 (1948).

${ }^{5}$ N. Wright, J. Opt. Soc. Am. 38, 69 (1948). since the source compartment has been mounted on a carriage moving on parallel ways.

The condenser and objective of our instrument are identical in construction, though only the former is shown in Fig. 1. The small convex mirror and the large concave mirror of the Schwarzschild system ${ }^{6}$ are

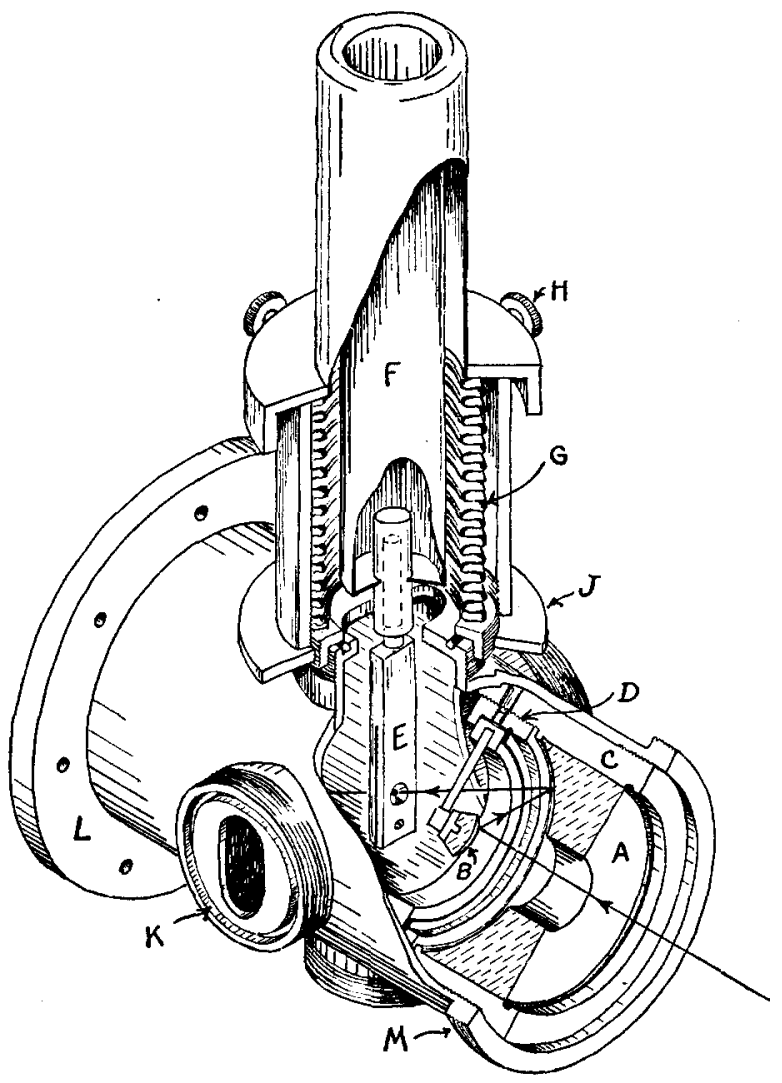

FIG. 1. Isometric drawing of the microilluminator showing portions cut away to indicate the internal construction. The path of an average ray is shown. $A$ and $B$ represent concave and convex mirrors carried by cell $C$, and subcell $D$. The sample holder $E$ projects from the Dewar thimble $F$. Lateral motion is provided by thumbscrews $H$, and vertical motion is permitted by the sylphon bellows $G$, which may be extended by rotation of threaded flange $J$. Grooves for " $O$ " rings, $K$, are provided on side arms and face of supporting flange $L . M$ is a threaded flange for attachment of polarizer.

'The optical path in similar systems is shown by R. B. Gore, Science 110, 510 (1949), and Blout, Bird, and Grey, J. Opt. Soc. Am. 40, 304 (1950). The optical design of these systems presumably differs somewhat from our own since entrance and exit beam are not parallel. 
mounted concentrically to eliminate coma, and the relative radii of curvature are chosen to minimize spherical aberration. The large mirror has a radius of curvature of $30 \mathrm{~mm}$, a diameter of $52 \mathrm{~mm}$, and an opening of $17 \mathrm{~mm}$ diameter at its center. The small mirror has a radius of $12 \mathrm{~mm}$ and a diameter of $11 \mathrm{~mm}$. Both mirrors are of aluminized glass. The focal length of the system is about $10 \mathrm{~mm}$, and the linear magnification obtained in connection with the IR-2 is about 18 . The numerical aperture is about 0.56 , and that of the obscuration is about 0.26 .

Before designing the optical system described, a careful exploration was made of the intensity distribution in the beam of our own spectrometer, and the final design was arrived at as a result of several compromises. In practice it is found that with optimum adjustment in the light path of the Beckman the microscope transmits as usable radiation approximately 40 percent of that available. The resolving power is completely adequate for the purposes for which it was intended, though naturally it is inferior to that obtainable with aspherical surfaces as in the Burch microscope ${ }^{7}$ used by Barer, Cole, and Thompson. ${ }^{1}$

The mounting of the optical parts should be evident in Fig. 1. The condenser and objective are mounted in cylindrical cells which slide, with a close fit, in the outer housing, except that both housing and cells are threaded for a short distance at the ends to provide for focusing. The convex mirrors are carried in subcells which screw into their housings to provide for accurate adjustment of the intermirror distance. The small mirror is carried by a "prong" extending from a ring which is held in the subcell by a "Truarc" retaining ring, and can be accurately centered by three screws, one of which is shown in the figure. The adjustment of the optical elements is very critical, both in respect to centering and to the distance between mirrors.

The sample holder is a copper bar, which at its upper end fits into a socket soldered to the inner thimble of a Dewar vessel of stainless steel. The lower end is divided into two prongs by a slot in which a silver chloride

\footnotetext{
${ }^{7}$ C. R. Burch, Proc. Phys. Soc. (London) 59, 41, 47 (1947).
}

plate bearing the specimen is clamped. The specimen is centered on conical apertures in the prongs, which serve as windows. It was found that considerable displacement of the sample holder occurs during the process of cooling so that it was necessary to provide means for relocating the sample at the optical focus. The Dewar vessel was consequently attached to the illuminator housing with a sylphon bellows. The threaded collar for adjustment of elevation, and two of the thumb screws providing lateral and longitudinal motion are shown.

Tests with a thermocouple indicate that the specimen, while illuminated, reaches a temperature of $-185^{\circ} \mathrm{C}$ about five minutes after the Dewar vessel is first cooled with liquid nitrogen.

The system has a reasonably high thermal economy. It requires about $50 \mathrm{ml}$ of liquid nitrogen to reach $-185^{\circ} \mathrm{C}$ and a consumption of about $40 \mathrm{ml} / \mathrm{hr}$ to maintain this temperature.

It was found necessary to employ a rather efficient vacuum system since condensation of ice on the specimen may otherwise occur rather rapidly, with consequent contamination of the spectrum. Using a silicone oil diffusion pump we are able to maintain a pressure of about $2 \times 10^{-5} \mathrm{~mm} \mathrm{Hg}$, under which condition the ice bands do not become serious during the first hour after cooling.

Although the instrument described was primarily designed for use with the Beckman IR-2 spectrometer, it has been successfully used with two other special spectrometers. One of these is a grating instrument with which observations were made using high resolution in the $3 \mu$ region. ${ }^{8}$ With another instrument having potassium bromide optics, measurements have been made down to $25 \mu$.

\section{ACKNOWLEDGMENT}

We gratefully acknowledge very useful advice given to us in the early stages of the optical design by Dr. J. L. Greenstein of this Institute.

${ }^{8}$ R. D. Waldron and R. M. Badger, J. Chem. Phys. 18, 566 (1950). 